José Alberdi

Lic. en Trabajo Social UNR. Magíster en Servicio Social. Docente de la Escuela de Trabajo Social (UNR). Trabajador Social del Centro Regional de Salud mental «Dr. Agudo Ávila»

Laura Coll

Lic. en Trabajo Social UNR. Trabajadora Social del Centro Regional de Salud mental «Dr. Agudo Ávila»
Eduardo Mutazzi

Psicólogo UNR. Doctor en Salud Mental. Psicólogo del Centro Regional de Salud mental «Dr. Agudo Ávila»

\section{Eleonora Vismara}

Lic. en Trabajo Social UNR. Trabajadora Social del Centro Regional de Salud mental «Dr. Agudo Ávila»

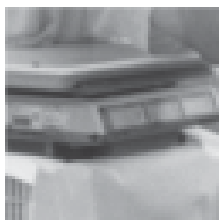

\title{
La Problemática de la Institucionalización Crónica y el Fenómeno de «Revolving Door» en Pacientes Usuarios del Centro Regional de Salud Mental Dr. Agudo Ávila (CRSM)
}

RESUMEN El objetivo fue realizar un análisis cuantitativo y cualitativo de la problemática de los pacientes de larga institucionalización (I.i.) o con repetición importante de internaciones en el CRSM. Para elaborar indicadores epidemiológicos en salud mental el estudio (analítico-descriptivo) se estableció sobre el total de pacientes de I.i. (45 pacientes, crónicos en la cultura institucional) mediante un corte longitudinal durante los meses de agosto/ septiembre de 2002.

Se debe destacar que del total de la muestra 25 pacientes $(55,5 \%)$ presentan I.i. siendo que, a 20 de ellos, $(44,5 \%)$ se los puede incluir en la categoría de «revolving door». El perfil construido de los pacientes arroja una tasa importante de institucionalización de pacientes con problemas de marginación socio-económica, así como un uso de la internación como forma de institucionalizar la exclusión (legal, social, familiar); se debe resaltar la institucionalización debido al déficit de otras políticas (discapacidad, tercera edad, etc.). En relación a los procesos de trabajo se observaron limitaciones en la organización del modelo asistencial debido a la hegemonía del dispositivo clínico que privilegia la atención de pacientes en crisis. Para la transformación del modelo manicomial (objeto de la ley de salud mental en vigencia) se hace necesario la promoción de servicios alternativos y sustitutivos.

PALABRAS CLAVES Epidemiología - Salud Mental - Reforma - Psiquiatría - Asistencia 


\section{Introducción}

El presente estudio desarrolla resultados parciales obtenidos a partir de la investigación epidemiológica analítica-descriptiva realizado para la elaboración de indicadores en salud mental en pacientes de larga institucionalización (I.i.) con la pretensión de fomentar una cultura de la evaluación de los procesos asistenciales a través de un perfil detallado de los grupos poblacionales. La presente investigación ha sido realizada por expreso pedido de la dirección del Centro Regional de Salud Mental Dr. Agudo Ávila (CRSM) constituyendo un equipo interdisciplinario para su ejecución.

- El diagnóstico de naturaleza cuantitativa y cualitativa implicó en la construcción de un instrumento metodológico que privilegió la siguiente información:

- Datos clásicos de naturaleza socio-demográfica (edad, estado civil, nivel de escolarización, cobertura social, trabajo, vivienda, etc.).

- Datos clínicos-asistenciales (registros historias clínicas, biografía asistencial, uso de medicamentos, estrategias asistenciales, etc.).

- Datos socio-relacionales (vínculos familiares, comunitarios y los efectos de la l.i., estigmatización).

- Principales necesidades y demandas para los cuidados formales y no formales de los pacientes para prevenir los riesgos de la internación (larga y/o breve).

Para la aplicación de dicho instrumento se conformó un equipo de trabajo interdisciplinario que realizó el trabajo de campo (relevamiento de datos) durante dos meses (agosto-septiembre 2002) registrando los datos en forma exhaustiva de la muestra seleccionada.

La principal fuente de información consultada fueron las historias clínicas, paralelamente a un trabajo de tipo más cualitativo de consulta a los equipos asistenciales, en especial se privilegió a los terapeutas de referencia (profesionales a cargo de la asistencia clínica o que acompañen o hayan acompañado el proceso asistencial del caso) y a los profesionales de enfermería, donde se privilegio la información específica de su área y referencias a la vida cotidiana del usuario en el Hospital.

Para la selección de la muestra se respetó lo acordado con los equipos en cuanto a la determinación del perfil o características de l.i. que dentro de la cultura institucional es considerado como "crónico" Es decir, la muestra quedó establecida con el total del universo de pacientes de l.i. de los cuales deberá destacarse un porcentaje que si bien no son pacientes de l.i. strictus sensu, son representantes de lo que en la literatura especializada considera como "revolving door" y que suponen una asistencia casi permanente para sostener su no hospitalización.

\section{Resultados}

En primer lugar se desarrollarán los datos cuantitativos generales de la muestra seleccionada. Se debe notar que los pacientes considerados crónicos por la cultura institucional o conceptualizados como de l.i. representan al momento del corte el $62,5 \%$ de las camas ocupadas.

A su vez se ha podido establecer que de ese total el 55,5\% puede ser considerado dentro de la categoría de "cronificado" y un 44,5\% pacientes de l.i. con experien- 
cias del fenómeno de "revolving door".

En relación con los anteriores resultados se puede decir que realizando un corte de las distintas décadas la población de l.i. se distribuye de la siguiente manera:

- el $4 \%$ fue institucionalizado en la década del ' 50 ;

- el 14\% tuvo su internación en la década del '80 prevaleciendo una tasa posterior a la apertura democrática;

- el $82 \%$ de la población de la muestra fue internada en la década del ' 90.

Al realizarse un cruce de datos entre la variable años de internación con las variables "crónico" y "revolving door" se elaboraron los siguientes datos:

- un $18 \%$ tiene características de l.i. sin ningún tipo de vínculo familiar ni social evidenciando lo que en general se entiende por cronificación por l.i.; sumado a este porcentaje se debe considerar una parte de la población internada en la década del ' 90 que es del $42 \%$. Por lo que se puede concluir que el $60 \%$ de la muestra no realizaba salidas periódicas, ni externaciones breves, así como no tenía prácticamente ningún tipo de participación en otras actividades intramuros;

- el 58 \% de la población internada en los años ' 90 es la que debe ser considerada como "revolving door" según lo que se ha expresado más arriba.

A partir de los siguientes datos es necesario establecer que proporcionalmente a la cantidad de años de internación mayores son los riesgos de cronificación (pérdida de lazos sociales y comunitarios, pérdida de la autonomía y cuidado de sí, etc.) sumado a esto debemos observar los riesgos que trae la internación sostenida por largos períodos ya que la población que corresponde a lo que hemos denominado "revolving door" va perdiendo progresivamente todo aquello que lo liga al "afuera" de la institución, corriendo el mismo riesgo que los clásicos crónicos, si no se crean servicios sustitutivos que puedan contener y apoyar las crisis subjetivas por fuera de la internación psiquiátrica.

\subsection{Características socio-demográficas de la población relevada}

En cuanto a la distribución por sexo de la muestra la mayoría esta compuesta por mujeres $(57,5 \%)$ y el $42,5 \%$ son hombres. De lo cual podemos conjeturar para futuras investigaciones el grado de vulnerabilidad de la mujer psiquiatrizada como puede ser rastreado en la abundante literatura sobre el tema.

Realizando un análisis sobre la composición etárea de la población de l.i. se puede observar que:

- el 13,5\% tiene más de 65 años;

- el $32 \%$ se encuentra entre los 50 y 65 años;

- el 38,5\% pertenece a la franja etárea comprendida entre los 35-50 años;

- el 16\% se encuentra entre los 20-35 años

Observando que gran parte de la población $(86,5 \%)$ corresponde a lo que el INDEC reconoce oficialmente como población económicamente activa y que se encuentra al momento de la internación sin trabajo por diversas problemáticas sociales más allá de su "patología subjetiva" y que el resto corresponde a problemáticas de tercera edad se deberá estar atento en cuanto al perfil o especificidad del CRSM.

Además debemos considerar que existe un porcentaje sugerente de la población que presenta problemas en relación a su Documento Nacional de Identidad (DNI, 
un tercio con problemas de extravío no resuelto, otro tercio en posesión de familiares, y el resto con el DNI en trámite) en particular y otras documentaciones esenciales.

En relación a la procedencia de los pacientes de l.i. se puede observar que los domicilios anteriores a la internación pertenecen prácticamente en su totalidad (90\%) al Departamento Rosario y utilizando la actual distribución geográfica del Programa de Descentralización Municipal de la Ciudad observamos que:

- 27\% corresponde al distrito Sur;

- 19\% al distrito Sur-Oeste;

- 13\% al distrito Centro;

- 10\% al Oeste;

- $5 \%$ al Noroeste;

- $9 \%$ al distrito Norte.

Se debe destacar la alta prevalencia de otras localidades del gran Rosario como Funes y Roldán (7\%), San Lorenzo (4,5\%), Villa Gdor. Galvez (11\%). Vale señalar a partir de las biografías asistenciales que encontramos en un $16 \%$ pacientes que han migrado del interior antes de su internación y con domicilio en Rosario.

El nivel educativo con que cuenta la población estudiada se distribuye de la siguiente manera:

- primario incompleto $24,5 \%$;

- primario completo 19\%;

- secundario incompleto $17 \%$;

- secundario completo 10\%;

- universitario incompleto 2,5\%;

- asistió a escuelas especiales el 9\%.

Se debe destacar que en el $18 \%$ de los casos no se registraron datos fidedignos sobre algún tipo de experiencia educativa. Estos datos describen a una población de bajo capital cultural siendo que aprox. sólo un $40 \%$ ha completado el nivel primario satisfactoriamente. Mediante las biografías asistenciales construidas se observa además que durante el tiempo de la hospitalización existieron mínimos accesos a algún tipo de capacitación laboral o educativa formal o protegida.

Uno de los principales problemas de los pacientes de l.i. resulta ser la falta de vivienda o con la imposibilidad de vivir en la vivienda familiar (nuclear o extendida) registrándose que:

- el $29 \%$ al momento del trabajo de campo no poseía vivienda ni familia directa que pudiera hacerse cargo;

- un 14\% posee vivienda familiar pero no cuenta con ningún tipo de apoyo por parte de los mismos;

- un $24 \%$ cuenta con vivienda familiar y con apoyo directo de su familia;

- un 33\% estaría en su derecho de habitar la vivienda familiar (casa paterna, materna y/o de anteriores matrimonios) pero en relación a las mismas se encuentran en situaciones de posesión conflictiva.

A partir del relevamiento podemos establecer las siguientes problemáticas en torno a la cuestión de las moradas: en primer lugar se destaca la "familia expulsiva"; en segundo, las "familias extensas con alto nivel de precariedad económica, hacinamiento, etc."; en tercer lugar, "situaciones de violencia familiar hacia el paciente o viceversa". Todo ello conduce a sostener la hipótesis de la labilidad del vínculo cuando existe sufrimiento psíquico sumada a situaciones de desempleo y desprotección 
social.

En lo que se refiere a la cobertura social de los pacientes (correlato que en parte se corroborará cuando se presenten los datos sobre situación laboral) se constata que:

- el $80 \%$ no contó, ni cuenta con ningún tipo de cobertura social;

- el $18 \%$ no cuenta con cobertura social pero tuvo en algún momento anterior a la internación en el CRSM (según consta en historias clínicas por internaciones anteriores y perdidas por desempleo o fallecimiento de los titulares);

- el $2 \%$ cuenta con cobertura social.

El acceso a algún tipo de beneficio de pensión por discapacidad o pensión especial en virtud de su situación se distribuye de la siguiente manera:

- un $40 \%$ de los pacientes tiene inicios de gestión previsional por parte de familiares que en su mayor parte como solución de compromiso con los terapeutas de referencia como forma de hacerse cargo de los cuidados y/o aumentar el nivel de autonomía económica de los pacientes;

- un $12 \%$ percibe una pensión siendo que la mitad de los mismos se encuentra administrada por sus familiares sin el debido contralor de sus usos y sin la seguridad que el beneficio recaiga sobre el paciente;

- un 7\% de los pacientes tienen familiares beneficiarios de subsidios transitorios (plan jefas y jefes).

Vale aclarar en este punto que ante la inexistencia de una pensión especial por la problemática de sufrimiento psíquico la única estrategia para ser beneficiario de algún subsidio resulta ser la declaración de discapacidad de los pacientes con sus consecuentes problemas de estigmatización y pérdida de derechos (por ejemplo en los casos de insania).

En lo que se refiere al apoyo familiar propiamente dicho (acompañamiento, cuidados informales, apoyo económico, visitas periódicas, visitas domiciliares, etc.) se ha observado que:

- un tercio de los pacientes tiene un apoyo familiar mínimo y está integrado parcialmente en el proceso asistencial;

- un segundo tercio no cuenta con ningún tipo de apoyo familiar pero que lo han recibido en sus primeros años de internación;

- el tercio restante cuenta con apoyo familiar en cuanto continúe institucionalizado sin interferir en sus vidas cotidianas.

Resulta imprescindible destacar que el grado de incorporación de la familia u otros lazos sociales depende en gran medida y es parcialmente gestado y producido por los equipos asistenciales (enfermeros, trabajadores sociales, psicólogos, psiquiatras, abogados).

La situación laboral de la población l.i. muestra que prácticamente la totalidad de los mismos se encuentra sin empleo y se observa además que:

- el $28 \%$ no posee ninguna experiencia laboral (incluye discapacitados);

- el 43,5\% tuvieron una experiencia laboral en el mercado informal de la economía (ventas callejeras, changas, oficios varios);

- un 16\% tuvo experiencia laboral en el mercado formal;

- el $20 \%$ de la muestra cumplió tareas de ama de casa y cuidado de los hijos;

- un $19 \%$ realizó tareas en el servicio doméstico.

En líneas generales pueden ser reconocidas tres tipologías de poblaciones en 
términos socio-económicos, utilizando para la construcción de las mismas la demarcación establecida por Castel en sus estudios sobre la cuestión social:

- en situación de vulnerabilidad en relación a la precariedad de sus vínculos de inserción socio-relacional (protecciones cercanas) y sus frágiles niveles de inserción socio-laboral anterior;

- en situación de desafiliación clásicas (baja o nula disponibilidad de soportes familiares, nula disponibilidad de cuidados e inserción social, en situación de exclusión estructural del mercado laboral);

- en situación de mayor integración social (siendo un porcentaje mínimo de pacientes), por capital cultural y económico anterior a su internación pero con escaso apoyo de sus familiares próximos a las estrategias de externación.

\subsection{Características de los vínculos sociales, niveles de autonomía y tipos de cuidados}

A partir de la re-construcción de las biografías asistenciales y del registro de las historias clínicas se ha podido establecer que con anterioridad a la internación o re-internaciones:

- un tercio de la muestra conseguía sostener relaciones y vínculos con vecinos, amigos y lazos comunitarios;

- otro tercio de los mismos presentaba dificultades con vecinos o amigos, encierros o aislamientos del espacio público y trasgresión sistemática de las normas mínimas de convivencia;

- el tercio restante de los internados lo ha sido por vía judicial de los colegiados de familia solicitado por familiares a instancias de vecinos o por abandono familiar.

Respecto de la vida cotidiana y el uso del tiempo libre se ha podido establecer a través del trabajo de campo que prácticamente la mitad de los pacientes de l.i. ha suspendido posteriormente a la internación la mayoría de sus intercambios sociales básicos (rutinización de los tiempos a la vida institucional: baño, comidas, mateadas, entrevistas esporádicas. Lo que transforma el tiempo libre u ocioso en una suerte de presente continuo). Durante el proceso de institucionalización solo una parte de la mitad restante se encuentra participando de tareas socio-asistenciales promovidas por la propia institución: talleres, centro cultural, taller de costura y ropería, fiestas y salidas grupales, venta de plantas, etc.). Es importante destacar que una parte de dicha población se sostiene con estrategias de sobrevivencia (contacto con los comercios vecinos, cuidado de autos, ventas en semáforos, pedidos y mangueos a transeúntes y automvilistas etc.) en los alrededores de la institución cuestiones que en el mejor de los casos generan una beneficencia informal y en la mayoría reafirman el estigma y su situación de vulnerabilidad social.

En lo que se refiere a los niveles de autonomía y capacidades de autocuidado de este grupo de pacientes se observa que:

- un tercio de los mismos tiene niveles de autonomía básicos que disminuyen en períodos de crisis necesitando en dicha situaciones de asistencia de terceros;

- otro tercio muestra, según lo consultado, necesitar para el ejercicio de su autonomía de un apoyo específico con prácticas de acompañamiento terapéu- 
tico;

- el resto de la población está caracterizada por lo que las categorías clásicas se encuentra como semidependiente o con dependencia total (problemas de control de esfínteres, víctimas de accidentes cerebro vasculares sin rehabilitación, discapacidad mental, tercera edad no autoválidos, deterioros clínicos, discapacidades motrices, etc.).

En cuanto a una síntesis de las trayectorias o biografías asistenciales de los pacientes y su concurrencia a instituciones sanitarias y psiquiátricas, se ha podido establecer una larga historia de contactos con la institución psiquiátrica resaltando que:

- un $43 \%$ fue asistido solamente por el servicio de prácticas ambulatorias (guardia y consultorio externo) del CRSM;

- un $18 \%$ proviene de asistencia por instituciones dedicadas específicamente

a la discapacidad;

- un 19\% tuvo largos períodos de internación en la Colonia Psiquiátrica de Oliveros;

- un $20 \%$ fue asistido por clínicas psiquiátricas privadas hasta la pérdida de su obra social.

En un párrafo aparte conviene destacar que en los pacientes con trayectoria de "revolving door" (44,5\% de la muestra) existe una baja utilización durante el tiempo de externación o alta de servicios ambulatorios de salud mental sean estos pertenecientes a servicios de hospitales generales o centros de APS demostrando el déficit de prácticas en salud mental alternativas y sustitutivas del manicomio, así como la falta de articulación o creación de una red de servicios provinciales y municipales en la que pueda tener un sustento.

A partir de la lectura de las historias clínicas y según el relato que en ellas aparece se pueden construir las siguientes descripciones de las principales manifestaciones subjetivas con o sin crisis de los pacientes de I.i:

- manifestaciones referidas como excitaciones psicomotrices;

- resaltan la presencia de síntomas delirantes y alucinatorios;

- presencia de fenómenos de agresividad (auto y hetero);

- conductas suicidas;

- extravíos y pérdidas de las nociones de tiempo y espacio;

- usuario de drogas, alcohol o uso abusivo de psicofármacos.

Es importante señalar que en un estudio posterior se deberá desarrollar este tópico pues la mayoría de las estrategias de control de dichas modalidades pasan por el aumento de medicación y la contención física como único recurso ante crisis o urgencias subjetivas.

En referencia a la elaboración de diagnósticos presuntivos se asocia un $54 \%$ a las psicosis, un $18 \%$ relacionados a problemas de uso de alcohol, drogas ilegales y abuso de psicofármacos, un $16 \%$ asociado a problemáticas de discapacidad (retraso mental, ACV, autismo, oligofrenia, etc.) y un $12 \%$ a neurosis graves.

En cuanto a las modalidades de la internación y basados en la ley 10772 se puede afirmar que, el $40 \%$ de la internaciones, no son voluntarias y en su mayoría remitidas por los juzgados de familia. Este punto invita a conjeturar sobre la rapidez que presentan las órdenes de internación en contrapartida a los procesos de externación o levantamiento de medidas en caso de altas. Resultando esto en un 
José Alberdi - Laura Coll - Eduardo Mutazzi - Eleonora Vismara

proceso iatrogénico que vulnerabiliza al paciente.

\section{Consideraciones principales y propuestas estratégicas}

Partiendo de este primer ejercicio de elaboración y creación de indicadores en salud mental serán señalados una serie de consideraciones válidas para aportar al proceso de debate tanto a nivel particular de la experiencia de reforma institucional y asistencial dirigidas por el equipo de internación como para el campo de reformas al modelo psiquiátrico tradicional y políticas en salud mental en general.

\subsection{La falta de una política consistente de reforma en salud mental}

Para la transformación del modelo manicomial (objeto de la ley provincial de salud mental 10772 sancionada y aprobada en 1991) se hace imperativa la existencia de servicios sustitutivos y alternativos al modelo manicomial clásico (art.17) así mismo como se vuelve esencial y central la articulación intersectorial entre el campo de las políticas sociales y de salud (ausente en la letra de la ley).

Ante la falta de lo que se ha expuesto se puede afirmar que la modalidad de desmanicomialización, a pesar del posicionamiento de los recursos humanos comprometidos en ese sentido, en la práctica se ha traducido en desasistencia por un lado y larga institucionalización por el otro, para esta afirmación se pueden consultar los datos referentes a los años de internación principalmente los de la década del '90 (coincidentes con la vigencia de la ley).

Esta situación corresponde centralmente a dos cuestiones relevantes:

- en primer lugar, debido a la actual «coyuntura» socio-económica que puso en crisis los sistemas clásicos de protección social y la fuerte fragmentación consecuente del tejido social. Situación ésta que afectó a la población en riesgo subjetivo de la siguiente manera: desocupación y precarización del empleo, desarticulación de los vínculos sociales, debilitamiento de los soportes de solidaridad y cuidados socio-familiares, precarización de la asistencia pública y cobertura social, deterioro de las funciones socio-familiares, fragilidad de los roles y funciones históricos en la estructura familiar, incapacidad de satisfacer las necesidades básicas principalmente de los que están en situación de vulnerabilidad, etc.;

- en segundo lugar, el desborde de la capacidad de los equipos al no cumplirse las disposiciones de la ley en salud mental vigente como la creación de diferentes dispositivos sustitutivos y alternativos de los grandes hospitales manicomiales como lo son centros de día, centro comunitarios de salud mental con camas de internación, internación en hospitales generales, servicios de acompañamientos terapéuticos, etc.

Se debe observar que los servicios de Salud Mental de los hospitales generales y los equipos de salud de los centros de atención primaria (APS) no alcanzan a instalarse como sustitutivos de la institucionalización psiquiátrica quedando en una función complementaria más que sustitutiva.

Esto sucede a pesar de las normativas provinciales en salud mental, debido a la falta de recursos (edilicios, humanos, económicos) que han impedido históricamente desarrollar un tipo de asistencia como el que recomienda la ley 10772, así 
como la falta de una política de salud mental que dirija este tipo de reformas necesarias. Por otro lado la especialización de los equipos de salud tanto de los hospitales generales como de los centros de APS están focalizados y capacitados para otro tipo de problemáticas subjetivas, no menos complejas, como neurosis, violencia familiar, crisis subjetivas asociadas a patologías orgánicas, etc.

Sumado a lo expuesto se debe tener en cuenta el déficit en la integración o creación de una red interinstitucional que aumente el grado de comunicación recíproca sobre todo para asegurar el seguimiento de los casos más vulnerables y frágiles a los efectos de prevenir las internaciones permanentes.

A su vez la persistencia del dispositivo de asistencia clínica clásico y hegemónico en el campo de la salud (frecuencias de tratamiento individual ambulatorio, medicación psicofarmacológica) se revela insuficiente para el abordaje de las problemáticas subjetivas y con "patología" del vínculo debiendo sustituirlas por prácticas o dispositivos de lo que se ha denominado en algunas reformas anti-manicomiales del contexto brasilero como de clínica ampliada.

\subsection{Los límites e iatrogenias de la internación psiquiátrica}

Un segundo eje de análisis es la importante tasa de institucionalización de personas con problemas de marginación social y económica revelando el uso de la internación como forma de institucionalizar la exclusión (legal, social, familiar) o de problemáticas que encuentra déficit en las políticas de su área (discapacidad mental, motriz, tercera edad).

A partir de los datos anteriormente expresados revela que la institucionalización psiquiátrica continúa siendo una descarga-ocultamiento de todo aquello que como sufrimiento, miseria, disturbio social resulta incoherente desde los códigos de interpretación culturales y desde los dispositivos de intervención de las instituciones sanitarias, sociales y jurídicas.

Sumado a esto se observa que la división clásica y crónica entre los servicios sanitarios y de promoción social impiden el aumento de autonomía y la protección de los pacientes así como la alternativa a la internación prolongada. Por ejemplo, los recursos con que cuenta actualmente el CRSM son básicamente para sostener la asistencia psicofarmacológica, ofertar psicoterapia y tratamiento ambulatorio con mínimos servicios sociales. Ante esta coyuntura queda el desafío para el departamento de trabajo social o los referentes asistenciales que cuentan con mínimas alternativas concretas para una oferta de cuidados y asistencia integral para el usuario y su familia.

Concurriendo con lo anterior se verifica claramente en un porcentaje de la muestra el déficit de las políticas de integración de las personas discapacitadas y las políticas sociales dedicadas a la tercera edad. La falta de centros de día y lugares de integración para discapacitados con niveles importantes de desarticulación familiar y el no cumplimiento de los convenios con las instituciones especiales del área, repite la problemática de la derivación a la deriva de otros servicios asistenciales o la depositación. Paralelo a eso se verifica un porcentaje menor de pacientes institucionalizados por estar sin domicilio fijo, reconocidos como "homelless" en la literatura especializada y finalmente se observa problemas en la persistencia de asistencia a población de tercera edad que la deberían cubrir otro tipo de políticas, verifi- 
José Alberdi - Laura Coll - Eduardo Mutazzi - Eleonora Vismara

cándose en los tres casos citados verdaderos agujeros negros en la asistencia, competencias entre instituciones por quién debe ser responsable y desasistencia de los casos más complejos y con menos recursos de protección cercana.

\subsection{El Caso del Suipacha y las metas del colectivo institucional}

Este último eje atañe a la singularidad del proceso de trabajo en el CRSM dado el perfil de servicios priorizado por este centro. Debido a la organización del trabajo centrada y hegemonizada para el paciente de reciente ingreso y en crisis subjetiva con internaciones breves la población de pacientes de l.i. se ve desplazada de las estrategias asistenciales.

Los desafíos presentados al colectivo institucional si pretende focalizar en la resolución de las principales necesidades y demandas de la población de larga institucionalización (crónicos y revolving door) dependerán, fundamentalmente, del aumento de presupuesto de recursos humanos y asistenciales y modificaciones estratégicas en las pautas de trabajo y la planificación de la tarea asistencial.

Como fue expresado la hegemonía del modelo de trabajo para pacientes en crisis no es suficiente ni eficaz para los pacientes de l.i. o para aquellos que necesiten acompañamiento terapéutico permanente para potenciar los niveles de autonomía, la problemática de la vivienda, los problemas de convivencia social, y principalmente su re-inserción familiar, social y comunitaria perdidas. Para ello se hace necesario un trabajo interdisciplinario que planifique las tareas de organización de redes de salud mental, dispositivos de acompañamiento y re-singularización de la asistencia

Paradojalmente la actual coyuntura conspira para el desenvolvimiento de este modelo de trabajo, siendo que las sobre-exigencias presentes en las demandas asistenciales del presente año revelan que durante los primeros ocho meses del corriente año hubieron 200 internaciones aproximadamente. Lo cual pone de manifiesto que de no existir un alto giro de cama y una asistencia privilegiada de esos casos "agudos" (remisión de la crisis y externación en breve lapso) se corren serios riesgos de que la manicomialización y los niveles de violencia institucional estén a la orden del día.

\subsection{Experimentando el cambio institucional: algunas ideas en el CRSM}

En un ejercicio de realismo utópico y a pesar de la evidente escasez histórica de recursos presupuestarios para la salud mental y en particular para los pacientes cronificados, se considera viable un conjunto de posibilidades que permitirían una mejora de la calidad asistencial, contando como diría Gastao Wagner de Souza Campos con la libertad y el compromiso de los trabajadores:

- Promover la conformación de un equipo de internación único e interdisciplinario que sustentado en el concepto de clínica ampliada formule una agenda de trabajo específica para los pacientes institucionalizados. Se debería incluir en el mismo personal de las áreas de mantenimiento, servicios generales y técnicos administrativos. Evitar la división clásica de equipos entre agudos y crónicos apuntando a un trabajo conjunto donde los referentes de los pacientes de I.i. colaboren con aquellos que estarán dedicados "full time" al trabajo con este 
tipo de pacientes.

- Que los abocados a la especificidad de atención y asistencia de este grupo de pacientes desarrolle una planificación estratégica en un programa de preparación de altas de pacientes I.i., teniendo en cuenta las problemáticas individuales y las compartidas por grupos de pacientes, notando resolver las necesidades básicas (vivienda, capacitación laboral, participación en actividades recreativas y de ocio, acompañamiento, etc.). En sí lo que sería la práctica de una clínica ampliada, reformulando y complejizando el tratamiento clásico de psicoterapia bajo la responsabilidad de su terapeuta de referencia, así como el control de medicación. Se recuerda que en esta planificación deberá constar la exigencia de financiamiento a las áreas de salud, promoción social, vivienda, educación y gerencia de empleo según corresponda como reparación de su injusto depósito en una institución pública.

- La priorización de la creación de un dispositivo de atención domiciliar y comunitario específico para el acompañamiento terapéutico y la contención de la crisis en el ámbito comunitario y familiar con el consecuente desarrollo presupuestario (recursos humanos, insumos, ambulancia, etc.). Fundamentalmente, para evitar las internaciones cuando fuere necesario y disminuir los riesgos de la larga hospitalización o los secuestros institucionales producidos por el ámbito judicial ante la falta de respuestas del campo de la salud mental. Evitando también la privatización del problema de los cuidados en la familia o en la comunidad de referencia, especialmente en los momentos más críticos, que es cuando se ven más fragilizados las seguridades familiares y comunitarias. - La designación de un presupuesto estable específico que permita generar alternativas asistenciales en la lógica del caso por caso sobretodo en aquellos pacientes que presentan niveles de autonomía importante pero que necesiten de acompañamiento para aumentar sus niveles de intercambio sociales y donde las alternativas de tipo más global no sean suficientes.

- Que la Dirección del CRSM con el apoyo y asesoramiento del equipo interdisciplinario de internación tenga como meta buscar la articulación de estrategias con otros dispositivos de la institución y extra-institucionales en pos de conformar una red de asistencia y cuidados en salud mental que priorice a la población institucionalizada. En especial ampliar el desarrollo de programas complementarios de vivienda, trabajo, educación, tiempo libre y cultura. Así como en la generación de convenios con ONGs e instituciones dedicadas a problemáticas cercanas que permitan el acogimiento de pacientes en instituciones culturales, vecinales, bibliotecas, que permitan disminuir las representaciones discriminatorias de la locura y constituirse en lugares de inclusión.

\section{Bibliografía}

ALMEIDA FILHO, N. "Epidemiología Social de los Desordenes Mentales" en: TUNDIS; COSTA (Comps.) Ciudadanía y Locura: Políticas de Salud Mental en el Brasil, Petropolis, Vozes, 1987.

AMARANTE, P. Psiquiatría Social y Reforma Psiquiátrica. Río de Janeiro, Fiocruz, 1994. 
DESVIAT, M. La Reforma Psiquiátrica. Los problemas de la Desinstitucionalización. Hospital de Leganes, España, 1990.

ROSANVALLON, P. La nueva era de las desigualdades. Buenos Aires, Manantial, 1997.

SCULL, R. Decarteration-Comunitty treatment and the deviant. Cambridge, Polite Press, 1984.

SOUZA CAMPOS, G. Gestión en salud. Buenos Aires, Lugar, 2001.

VASCONCELOS, E. "Evaluación de servicios y revolving door" en: Revista Transversiones: Salud Mental, Desinstitucionalización y Abordajes Psicosociales. Río de Janeiro, UFRJ, 2000.

. "Servicio Social e Interdisciplinariedad. El ejemplo de la Salud Mental" en: Revista Servicio Social y Sociedad n 54, San Pablo, Brasil, 2000. 